\title{
First-principles investigation of armchair stanene nanoribbons
}

\author{
M. Fadaie ${ }^{a, b}$, N. Shahtahmassebi ${ }^{a}$, M.R. Roknabad ${ }^{a}$, O. Gulseren ${ }^{b, *}$ \\ a Department of Physics, Ferdowsi University of Mashhad, Mashhad, Iran \\ ${ }^{\mathrm{b}}$ Department of Physics, Bilkent University, Ankara, Turkey
}

\section{A R T I C L E I N F O}

\section{Article history:}

Received 4 May 2017

Received in revised form 30 October 2017

Accepted 17 November 2017

Available online 22 November 2017

Communicated by R. Wu

\section{Keywords:}

2D materials

Stanene

Nanoribbons

Optical properties

Electronic structure

Density functional theory

\begin{abstract}
A B S T R A C T
In this study, we systematically investigated the structural, electronic and optical properties of armchair stanene nanoribbons (ASNRs) by using the first-principles calculations. First, we performed full geometry optimization calculations on various finite width ASNRs where all the edge $\mathrm{Sn}$ atoms are saturated by hydrogen atoms. The buckled honeycomb structure of two dimensional (2D) stanene is preserved, however the bond length between the edge Sn atoms is shortened to $2.77 \AA$ compared to the remaining bonds with $2.82 \AA$ length. The electronic properties of these nanoribbons strongly depend on their ribbon width. In general, band gap opens and increases with decreasing nanoribbon width indicating the quantum confinement effect. Consequently, the band gap values vary from a few meV exhibiting lowgap semiconductor (quasi-metallic) behavior to $\sim 0.4-0.5 \mathrm{eV}$ showing moderate semiconductor character. Furthermore, the band gap values are categorized into three groups according to modulo 3 of integer ribbon width $N$ which is the number of Sn atoms along the width. In order to investigate the optical properties, we calculated the complex dielectric function and absorption spectra of ASNRs, they are similar to the one of 2D stanene. For light polarized along ASNRs, in general, largest peaks appear around $0.5 \mathrm{eV}$ and $4.0 \mathrm{eV}$ in the imaginary part of dielectric functions, and there are several smaller peaks between them. These major peaks redshifts, slightly to the lower energies of incident light with increasing nanoribbon width. On the other hand, for light polarized perpendicular to the ribbon, there is a small peak around $1.6 \mathrm{eV}$, then, there is a band formed from several peaks from $5 \mathrm{eV}$ to $\sim 7.5 \mathrm{eV}$, and the second one from $8 \mathrm{eV}$ to $\sim 9.5 \mathrm{eV}$. Moreover, the peak positions hardly move with varying nanoribbon width, which indicates that quantum confinement effect is not playing an essential role on the optical properties of armchair stanene nanoribbons. In addition, our calculations of the optical properties indicate the anisotropy with respect to the type of light polarization. This anisotropy is due to the quasi-2D nature of the nanoribbons.
\end{abstract}

(c) 2017 Elsevier B.V. All rights reserved.

\section{Introduction}

In recent years, graphene, first example of two dimensional (2D) materials, is attracting a lot of attention because of its exotic properties. Besides various nanostructures based on graphene, one-dimensional (1D) nanoribbons has been thoroughly explored. These 1D nanostructures exhibit interesting properties dependent on their size [1-3], topological shapes [4], geometry [2,5] and edge-passivation pattern [6,7]. The electronic structure of the graphene nanoribbons is extensively investigated; because of the finite width, quantum confinement effect leading to band gap opening is essential in graphene nanoribbons [8,9], and it has shown that band gaps are inversely proportional to the ribbons width [10]. In previous studies, armchair graphene nanoribbons

\footnotetext{
* Corresponding author.

E-mail address: gulseren@fen.bilkent.edu.tr (0. Gulseren).
}

(AGNRs) are divided into three groups according to the modulo 3 of the number of rows of the carbon atoms along the nanoribbon edge, so these groups are labeled with $3 M+1,3 M$ and $3 M-1$ where $M$ is an integer. The electronic properties of these families and the gap openings of them strongly depend on the ribbon width [11-13]. The armchair graphene nanoribbons are metallic if the number of rows of carbon is $3 M-1$ while the AGNRs falling into $3 M$ and $3 M+1$ family are semiconductor [7]. On the other hand, zigzag graphene nanoribbons are always metallic, however transverse electric field can be used to open and control their band gap $[14,15]$. Moreover, the zigzag graphene nanoribbons display remarkable edge ferromagnetism [16].

Aside from numerous both theoretical and experimental studies on the properties of graphene nanoribbons, there is a considerable effort to synthesize and to investigate various types of 1D nanostructures apart from GNRs. For instance, Cahangirov et al. investigated the nanoribbons from 2D silicene which is formed by silicon 
atoms, one row below the $C$ of group IV of periodic table, analogous to graphene. They have shown that electronic and magnetic properties of armchair nanoribbons of silicene are strongly dependent on their width. Similar to the graphene nanoribbons, silicene nanoribbons are also sorted into three groups. Silicene nanoribbons (SNRs) falling in $3 M+1$ family and 'low-width' ribbons of $3 M$ family are semiconductors, otherwise all the $3 M-1$ group SNRs have band gaps less than $0.1 \mathrm{eV}$, so they are low-band gap semiconductors, or quasi-metallic in practice. Comparison of the band gap variation of silicene and graphene nanoribbons shows that GNRs in general have larger band gaps [7]. Further to these inspiring theoretical predictions, experimental studies carried out by Nakano et al. reported the soft synthesis of a single Si monolayer sheet over a substrate, holds the promise for the synthesis of Si honeycomb nanoribbons $[17,18]$.

In group IV of the periodic table, germanium comes down under the carbon and silicon, so one wonders the possibility of 2D structure from Ge atoms. In a following study, the armchair and zigzag nanoribbons from the low-buckled germanene have been studied by Cahangirov et al., and reported that these ribbons can exhibit fascinating electronic and magnetic properties [19]. Tin is the next group IV element under Ge in the periodic table. Stanene is a new 2D hexagonal material which is made of $\mathrm{Sn}$ atoms and it has been synthesized recently [20]. There are some very recent works that investigate the structural and mechanical properties of this new 2D nanostructure $[21,22]$.

The purpose of the present work is study of the structural, electronic and optical properties of armchair stanene nanoribbons of widths ranging from $N=7$ to $N=17$ atomic rows employing firstprinciple simulations based upon density functional theory. In this paper, we first described the details of computational parameters which were used and the supercell model employed for the calculations. Then, we reported the structural and electronic properties of ASNRs, and discussed their band gap variation. Finally, the optical properties for two direction of light polarization are studied.

\section{Computational method}

All calculations were conducted using Siesta code based on density functional theory (DFT). We employed the generalized gradient approximation (GGA) within the Perdew-Burke-Ernzerhof (PBE) formalism [23] for the exchange-correlation potential in our calculations. Because of the periodic boundary conditions, a supercell geometry was adopted in order to avoid the spurious interaction between isolated nanoribbons, a large vacuum spacing of at least $20 \AA$ for both layer-layer and edge-edge separations are introduced. Stanene nanoribbons were placed in the $y z$-plane. The Brillouin zone integrations were performed using $1 \times 20 \times 1 k$-point mesh within the Monkhorst-Pack scheme and cutoff energy of $950 \mathrm{eV}$ (70 Ryd) was used after extensive test calculations with respect to convergence. All dangling bonds of the edge $\mathrm{Sn}$ atoms were passivated with $\mathrm{H}$ atoms. Then, all atomic positions were fully relaxed using a conjugate gradient technique. During these geometry optimizations, all the internal coordinates were relaxed until the Hellmann-Feynman forces were less than $0.005 \mathrm{eV} \AA^{-1}$.

In order to investigate the optical properties of stanene nanoribbons, we have calculated the complex frequency dependent dielectric function as well. The absorption spectra can be obtained by considering the transitions from occupied to unoccupied states within the first Brillouin zone. Dielectric function calculations in SIESTA are based on the first order time dependent perturbation theory. To this end, first the self-consistent ground-state DFT energies and eigenfunctions are computed and then they are used to calculate the dipolar transition matrix elements. Thus, the imaginary part of the dielectric function pinpoint the optical absorption which is essentially calculated from the transition rate between valance and conduction band states.

We only need the calculation of imaginary part of the complex dielectric function which is expressed as sum of real and imaginary parts, $\varepsilon(\omega)=\varepsilon_{1}(\omega)+i \varepsilon_{2}(\omega)$. Within the dipole approximation, the imaginary part, $\varepsilon_{2}(\omega)$, is given by the following expression:

$$
\begin{aligned}
& \varepsilon_{2}(\omega) \\
& =\frac{e^{2}}{\pi m^{2} \omega^{2}} \sum_{\nu, c} \int_{B Z} d \vec{k}\left|\left\langle\psi_{c k}|\hat{e} \cdot \vec{p}| \psi_{\nu k}\right\rangle\right|^{2} \delta\left(E_{c}(k)-E_{v}(k)-\hbar \omega\right)
\end{aligned}
$$

where the sum runs over every pair of valance (filled) and conduction (empty) band states and the integral is over all $k$-points in the Brillouin zone, $c$ and $v$ letters refer to the conduction and the valence band states respectively. $E_{(c, v)}(k)$ and $\psi_{(c, v), k}$ are the corresponding energy and eigenfunction of these states. The electronic dipole transition matrix element is between the pair of filled and empty states where $\hat{e}$ is the polarization vector and $\vec{p}$ is the momentum operator.

Having the imaginary part, $\varepsilon_{2}(\omega)$, allows the calculation of the real part of the complex dielectric function from causality, i.e. the Kramers-Kronig relations:

$\varepsilon_{1}(\omega)=1+\frac{2}{\pi} P \int_{0}^{\infty} \frac{\varepsilon_{2}\left(\omega^{\prime}\right) \omega^{\prime}}{\omega^{\prime 2}-\omega^{2}} d \omega^{\prime}$

where $P$ denotes the principle part. Then, all optical constants can be calculated from the complex dielectric function once the band structure of the system is known [24]. Eq. (1) is the basic expression which connects the band structure to the optical properties. For example, the absorption coefficient $\alpha(\omega)$ is

$\alpha(\omega)=\frac{\omega}{c n(\omega)} \varepsilon_{2}(\omega)$

where $n$ is the refractive index which can be calculated from $\varepsilon_{1}(\omega)$ and $\varepsilon_{2}(\omega)$, and $c$ is the speed of light. In most of the practical cases, the peaks and the structure of $\varepsilon_{2}(\omega)$ and $\alpha(\omega)$ are rather similar.

In the present work, dielectric functions are calculated for two different direction of light polarization, along ASNRs and perpendicular to stanene nanoribbons and also for unpolarized light. Dielectric function $\varepsilon(\omega)$ is calculated in the energy interval from 0 to $15 \mathrm{eV}$. For these calculations, a denser $k$-point mesh, i.e. $1 \times 200 \times 1$, within the Monkhorst-Pack scheme is used for the Brillouin zone integrations.

\section{Results and discussions}

\subsection{Structural and electronic properties}

Fig. 1(a) shows the top and side views of optimized 2D hexagonal sheet of stanene. In our previous work [22], we calculated its lattice parameter as $a=4.68 \AA$ and buckling height as $d_{0}=0.86 \AA$, which are in a good agreement with other studies [21,25]. The armchair stanene nanoribbon structures are prepared from this infinite quasi-planar structure by keeping only $N$ dimer lines along the ribbon direction which extends to infinity, imposed by periodic boundary condition along this direction. As indicated in Fig. 1(b), the ribbon width is shown by $W_{a}$ and the buckling in the ribbons is labeled by $d$. Armchair type of bond structure appears along the both edges of the ribbon. All the edge Sn atoms are saturated by $\mathrm{H}$ atoms to avoid the effects of dangling bonds. In our calculations, we consider ASNRs with different widths, labeled by $N$ as NASNR, and $N$ is varied from 7 to 17 . For example, the schematic structure of the 13ASNR is shown in Fig. 1(b) from top and side. 

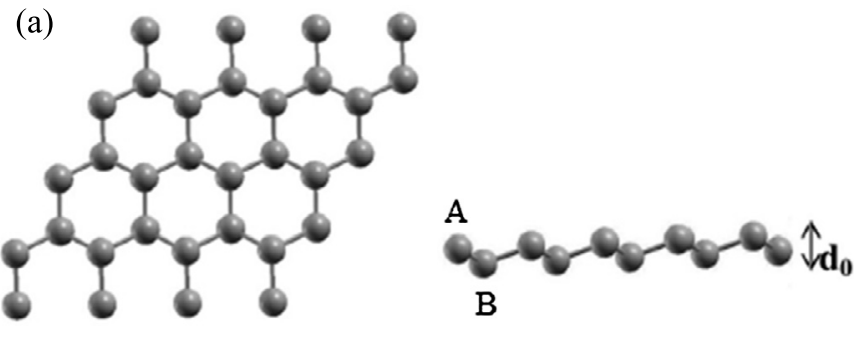

(b)

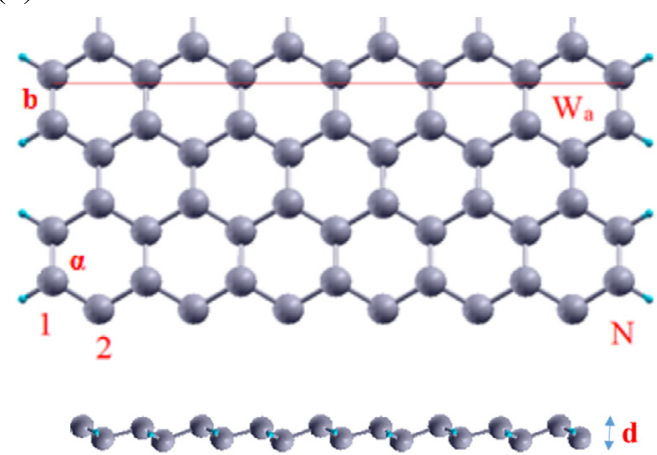

Fig. 1. Top and side view of (a) a 2D stanene layer, (b) 13-armchair stanene nanoribbon. The gray (large) and blue (small) balls represent tin and hydrogen atoms, respectively. The ribbons are periodic along the ribbon direction. The ribbon width and buckling are denoted by $W_{a}$ and $d$, respectively. (For interpretation of the references to color in this figure legend, the reader is referred to the web version of this article.)

Examining the geometrical structure of ASNRs shows that the length of bonds in edges are shorter than the other bonds. Namely, the length of edge bond, shown by "b" in the Fig. 1(b), is about $2.77 \AA$ while the length of rest of the bonds are $2.82 \AA$. The buckling in nanoribbons is also smaller compared to the $2 \mathrm{D}$ infinite structure. For example, it is $0.63 \AA$ for 7ASNR, and it increases with increasing ribbon width. There are some differences in the bond angle values as well. While the bond angles are almost uniform with the value $112.62^{\circ}$ in $2 \mathrm{D}$ stanene structure, the edge bond angles, " $\alpha$ " in Fig. 1(b), are larger than the other bond angles in nanoribbons, and it is smaller than the 2D stanene angles. The edge bond angles are as small as $111.04^{\circ}$ in 7ASNR, while the other ones around $109^{\circ}$, and they both increase with increasing nanoribbon width, eventually approaching $2 \mathrm{~d}$ stanene values.

Next, we continue by the electronic properties. The computed electronic band structures of some armchair stanene nanoribbons in the absence of spin orbit coupling are presented in Fig. 2. In all band structure plots, the Fermi level is shifted to zero. As seen on Fig. 2, all the bands folded between $\Gamma$ and $X$ points according to the ribbon width have both valance band maximum and conduction band minimum at $\Gamma$ point, hence exhibit direct band gap semiconductor character. Nanoribbon width increases going from top panel to down and from left to right panel in Fig. 2, obviously, the number of bands increases with increasing ribbon width. Furthermore, the band gap values are categorized into three groups according to modulo 3 of integer ribbon width $N$ which is the number of $\mathrm{Sn}$ atoms along the width. We named these three group as $N=3 M+1, N=3 M-1$ and $N=3 M$, where $M$ is an integer. In Fig. 2, the band structures displayed in the same row belong to same group, and the order of the rows follow the above sequence.

The elaborate further, the variation of the band gap as a function of ribbon width is presented in Fig. 3. All band gaps are direct and the band gap values vary from a few meV, exhibiting low-gap semiconductor (or quasi-metallic) behavior, to $\sim 0.4-0.5 \mathrm{eV}$, show-
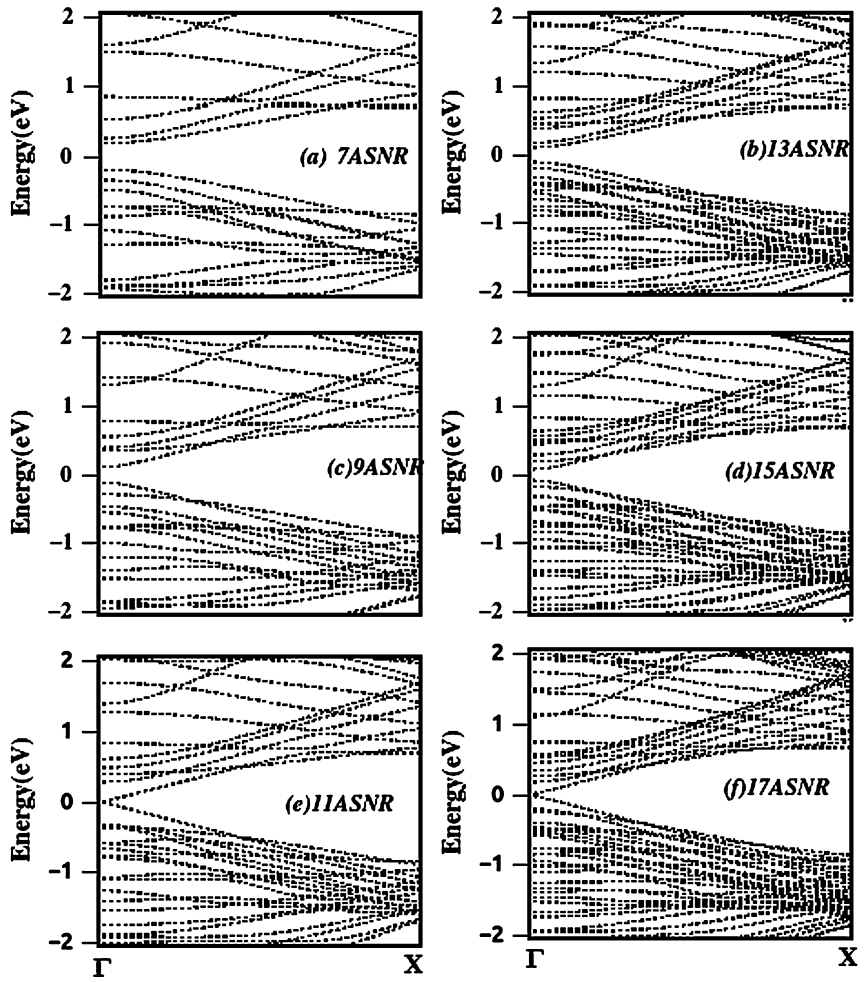

Fig. 2. Electronic band structures of various armchair stanene nanoribbons.

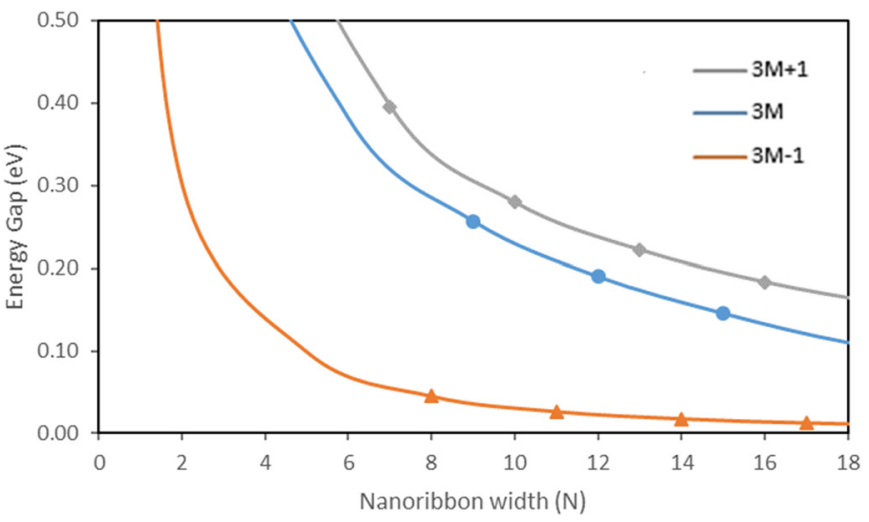

Fig. 3. Variation of band gap as a function of ribbon width.

ing moderate semiconductor character. After all, the band gap exhibits an oscillatory behavior as a function of ribbon width. However, as discussed above, ASNRs band gaps can be separated into three groups according to modulo three of ribbon width $N$. Within each family, the band gap decreases as the width of nanoribbons increases, which indicates the quantum confinement effect. By comparing the band gap variation of ASNRs and AGNRs, it is concluded that ASNRs have smaller band gaps [7].

The variation of the band gap within each group is smooth, and there is an inverse width dependence. We could find a formula to describe each family by fitting the calculated band gap values. These formulas present the correct asymptotic behavior and they might be used to calculate directly the bandgap for every width of nanoribbons, therefore provide a qualitative information of the electronic structure of nanoribbons beyond this range of widths. The calculated band gaps can be described by inverse power dependence of ribbon width as:

$E_{g}=a N^{-b}$ 
Table 1

Values of $a$ and $b$ for each family of nanoribbons.

\begin{tabular}{lll}
\hline Family & $a(\mathrm{eV})$ & $b$ \\
\hline $3 M+1$ & 2.3527 & 0.9205 \\
$3 M$ & 3.6354 & 1.2149 \\
$3 M-1$ & 1.4489 & 1.6694 \\
\hline
\end{tabular}

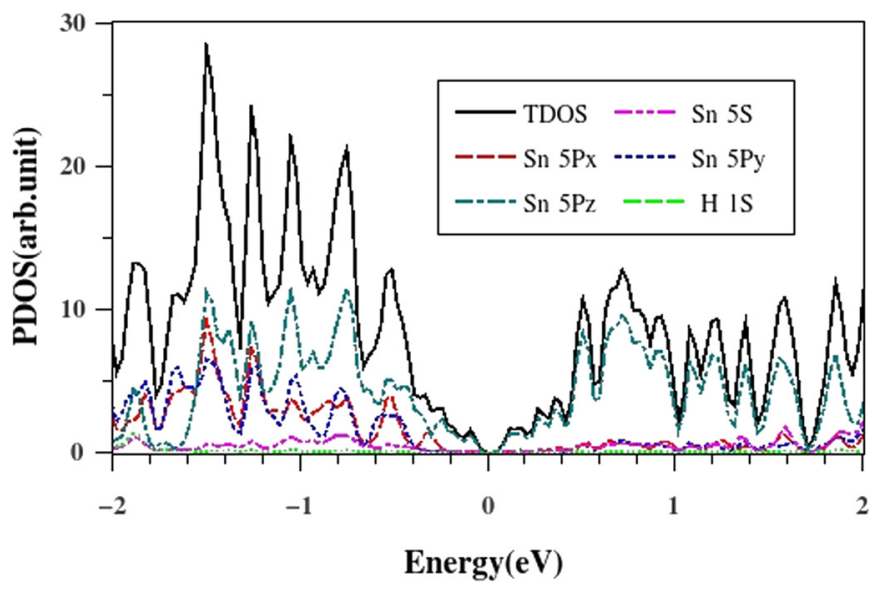

Fig. 4. Total and projected density of states (PDOS) of 13ASNR.

where $a$ and $b$ are two fitting parameters and they are different for each group. The values are shown in Table 1. Solid curves in Fig. 3 are drawn by these parameters and they exhibit an excellence representation of the band gap values.

In order to understand the characteristics of states around Fermi energy, total and projected densities states (PDOS) of 13 armchair stanene nanoribbons is represented in Fig. 4 as an example. As seen in Fig. 4, both valance and conduction band edges have $\mathrm{Sn} 5 p_{z}$ orbital character. This extends over the conduction band states up to $2 \mathrm{eV}$ shown in the Fig. 4. However, for the valance band states, from $0 \mathrm{eV}$ to $-1.8 \mathrm{eV}$ shown in the figure, although the main contribution is from the Sn $5 p_{z}$ orbitals, there is an appreciable contribution from the $\operatorname{Sn} 5 p_{x}$ and $5 p_{y}$ orbitals as well.

\subsection{Optical properties}

Understanding the optical spectra is essential for optoelectronic applications and it is one of simple but powerful characterization tool for structures. In order to investigate the optical absorption, we have calculated the dipole matrix elements between occupied and empty states for each case, which is essentially the imaginary part of the dielectric function, $\varepsilon_{2}(\omega)$. We calculated the optical spectra employing first order time-dependent perturbation theory as described in methods section. The complex and frequencydependent dielectric function describes how light interacts when propagating through matter. Essentially, its real part indicates the dispersion effects while the imaginary part describes the absorption.

The real and imaginary part of the complex dielectric function are calculated for two different polarization of light, along ASNRs and perpendicular. Fig. 5 shows the imaginary part of dielectric function of selected ASNRs. In all figures curves are shifted in vertical axis for clear presentation. $\varepsilon_{2}(\omega)$ calculated both for parallel and perpendicular polarizations are similar to the imaginary part of dielectric function of 2D stanene [22].

For light polarized along ASNRs, $\varepsilon_{2}(\omega)$ exhibits two main peaks around $0.5 \mathrm{eV}$ and $4.0 \mathrm{eV}$ and there are several smaller peaks between them. With increasing nanoribbon width, these major peaks redshifts, slightly to the lower energies of incident light. The first
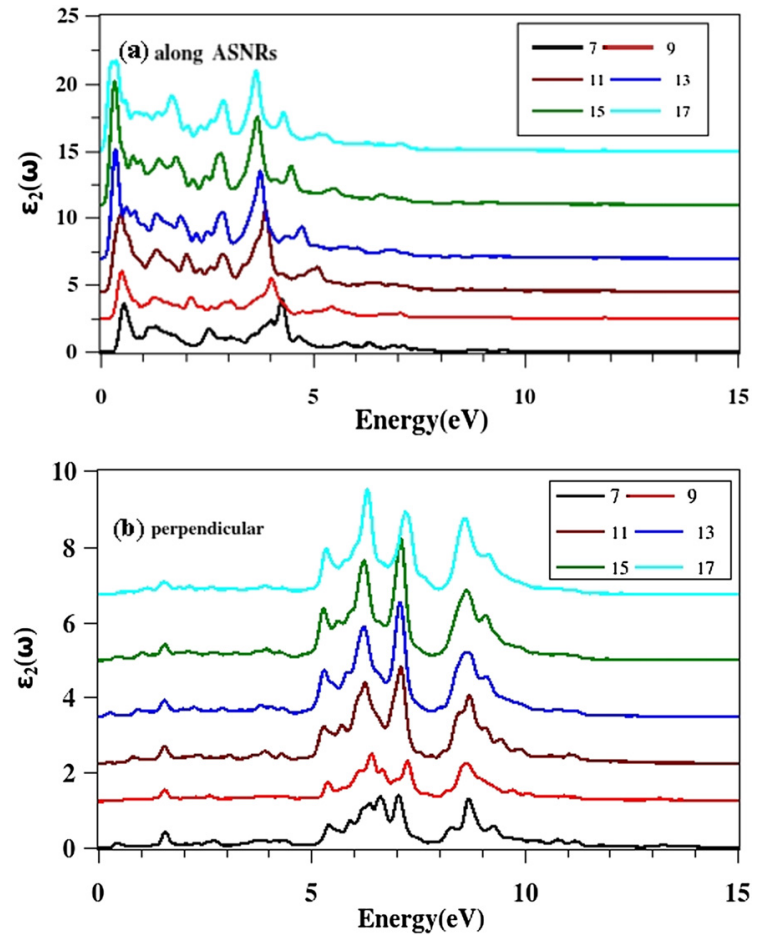

Fig. 5. The imaginary part of dielectric function of armchair stanene nanoribbons; light polarization is (a) along ASNRs, (b) perpendicular to the ribbons plane. Lines are shifted along the vertical axis, from lowest $M$ to the highest one, for separating the curves.

peak is from the transition from the states around valance band maximum to the conduction band edge, since it appears on the onset of the band gap. So, the redshift of this peak follows the variation of the band gap with ribbon width. Second major peak around $4 \mathrm{eV}$ is broader for small ribbon width, however it sharpens with increasing width. The redshift is also larger for this peak compared to the first one. In between these two peaks, from several smaller peaks two of them become dominant around 2 and $3 \mathrm{eV}$ with increasing ribbon width.

For light polarized perpendicular to ribbon plane, as presented in Fig. 5b, features of calculated $\varepsilon_{2}(\omega)$ are different compared to in plane polarization along the ASNRs case. For all the ASNRs studied here from $N=7$ to $17, \varepsilon_{2}(\omega)$ is very small up to $5 \mathrm{eV}$, there is only a small peak around $1.6 \mathrm{eV}$. Then, there are two energy windows where two broad bands are observed. First, there is a band formed from several peaks from $5 \mathrm{eV}$ to $\sim 7.5 \mathrm{eV}$, then the second one from $8 \mathrm{eV}$ to $\sim 9.5 \mathrm{eV}$. In contrast to the polarization along the ribbon case, peak positions hardly move with varying nanoribbon width, which indicates that quantum confinement effect is not playing an essential role for these transitions in perpendicular polarization. With increasing ribbon width, linewidth of the peaks especially around 6.5 and $7 \mathrm{eV}$ decrease.

Next, we presented the real part of the dielectric function, $\varepsilon_{1}(\omega)$, which was calculated from $\varepsilon_{2}(\omega)$ by using the KramersKroning [26] relation for both direction of light polarizations. In both cases, there are dips in the amplitude which some of them attain small negative value. The negative values are seen in the range of 3.5-6.0 eV for polarization along the ASNRs and at $7 \mathrm{eV}$ for polarization perpendicular to the ribbons. These negative values indicate the plasmonic excitations. For polarization along the ASNRs, the effect of ASNRs' width variations are seen mostly at low frequencies of incident electromagnetic wave. It is also observed that the static dielectric constant (real part of dielectric function's value at zero energy) increases with increasing width of ASNRs (Fig. 6). 

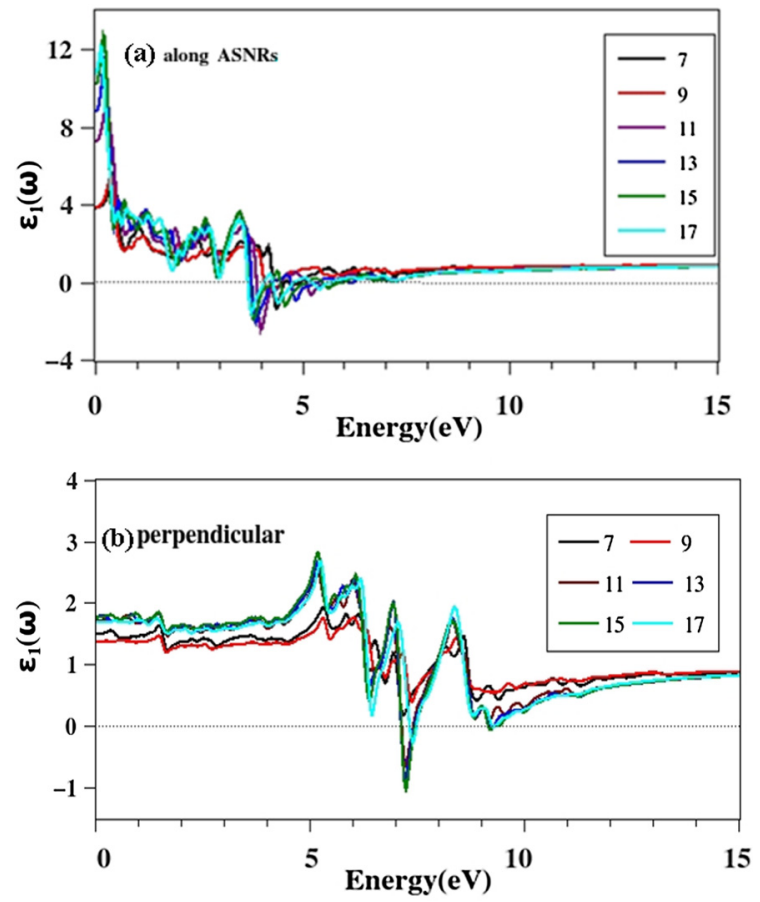

Fig. 6. The real part of dielectric function of armchair stanene nanoribbons; light polarization is (a) along ASNRs, (b) perpendicular to the ribbons plane.

The optical absorption spectra presented in Fig. 7 were calculated from the complex dielectric function for polarization perpendicular and along the ASNRs and also for unpolarized light.

It is clear that all features of $\varepsilon_{2}(\omega)$ and $\alpha$ are very similar which can be confirmed by comparing the imaginary part of the complex dielectric function shown in Fig. 5 and the optical absorption coefficient displayed in Fig. 7. First of all, it is evident that the optical properties of ASNRs are anisotropic with respect to the type of light polarization from the comparison of Fig. 7(a) and (b). The differences between the two polarizations are a consequence of the selection rules raised from the electronic dipole transition matrix elements. While the absorption coefficient exhibits features up to $7 \mathrm{eV}$, mostly dominant around $4-5 \mathrm{eV}$ for polarization along the ASNRs, it is almost zero till $5 \mathrm{eV}$, and then a broad feature between 5 to $10 \mathrm{eV}$ with five major peaks at around 5.5, 6.5, 7, 9 and $9.5 \mathrm{eV}$ for polarization perpendicular to the ribbon. The absorption coefficients for unpolarized light are presented in Fig. 7(c). The anisotropy in $\alpha$ is reflected in this total, while the features below $5 \mathrm{eV}$ look like the ones of in-plane polarization, the ones above the $5 \mathrm{eV}$ are similar to the perpendicular polarization ones. As we discussed earlier, the band gap opens and increases with increasing ribbon width while their value oscillates according to modulo 3 of integer ribbon width. The zero value of the absorption coefficient at the beginning of the spectrum is correlated with this. Significantly, since the shape and peak positions are almost independent from the ASNR width, we can conclude that the quantum confinement has no significant effect on the optical properties of armchair stanene nanoribbons. These universal optical properties, which are independent of the size of the nanostructures, have also been demonstrated in $\mathrm{ZnO}$ nanostructures as well $[27,28]$.

\section{Conclusions}

In summary, we have studied the structural, electronic and optical properties of armchair stanene nanoribbons by using the DFT based calculations. The structural investigations show some differences in bond lengths and angles which are related to the edge atoms. ASNRs show a typical three family variation of band
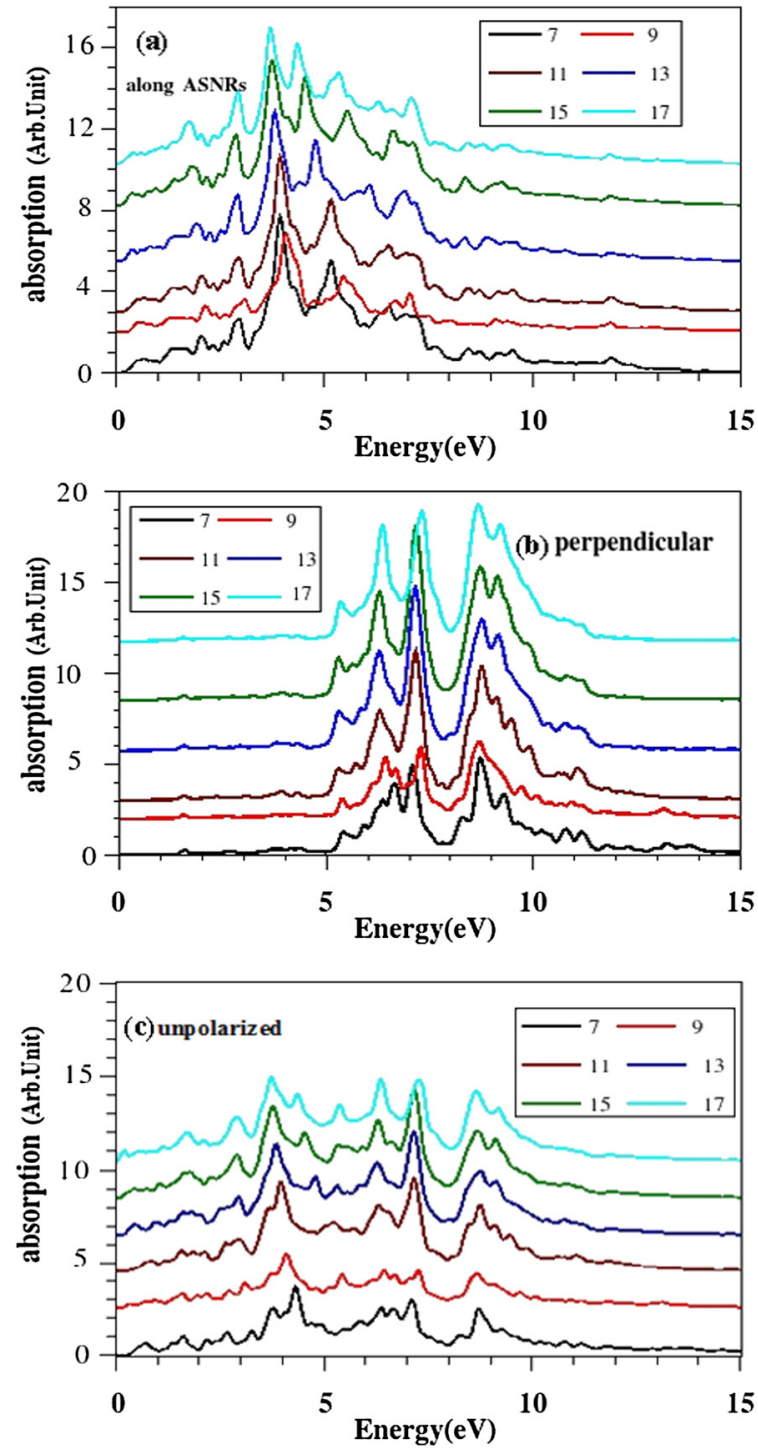

Fig. 7. Absorption spectra of ASNRs for polarization of light (a) along ASNRs, (b) perpendicular to the ribbons plane, (c) unpolarized. Lines are shifted along the vertical axis, from lowest $M$ to the highest one, for separating the curves.

gap with width. We labeled these families with $3 M, 3 M+1$ and $3 M-1$, where $M$ is an integer. The electronic properties of these families strongly depend on their ribbon width. Within each group, band gap decay with increasing width.

Our results for optical investigations show the similarity between dielectric function spectra of ASNRs and stanene. For light polarized along the ASNRs, $\varepsilon_{2}(\omega)$ exhibits two main peaks around $0.5 \mathrm{eV}$ and $4.0 \mathrm{eV}$ and there are several smaller peaks between them. These major peaks redshifts, slightly to the lower energies of incident light with increasing nanoribbon width. For perpendicularly polarized light, there is only a small peak around $1.6 \mathrm{eV}$ in $\varepsilon_{2}(\omega)$. Then, there are two energy windows where observed a broad band. First, there is band formed from several peaks from $5 \mathrm{eV}$ to $\sim 7.5 \mathrm{eV}$, then second one from $8 \mathrm{eV}$ to $\sim 9.5 \mathrm{eV}$. Due to the insensitivity of the shape and peak positions in nanoribbon width, we can conclude that the quantum confinement has no significant effect on the optical properties of armchair stanene nanoribbons. In addition we observed an anisotropy with respect to the type of light polarization. 


\section{Acknowledgement}

OG acknowledges the support from Scientific and Technological Research Council of Turkey (TUBITAK-115F024).

\section{References}

[1] K. Nakada, M. Fujita, G. Dresselhaus, M.S. Dresselhaus, Edge state in graphene ribbons: nanometer size effect and edge shape dependence, Phys. Rev. B, Condens. Matter 54 (1996) 17954

[2] V. Barone, O. Hod, G.E. Scuseria, Electronic structure and stability of semiconducting graphene nanoribbons, Nano Lett. 6 (2006) 2748.

[3] L. Yang, C.-H. Park, Y.W. Son, M.L. Cohen, S.G. Louie, Quasiparticle energies and band gaps in graphene nanoribbons, Phys. Rev. Lett. 99 (2007) 186801.

[4] Y.W. Son, M.L. Cohen, S.G. Louie, Energy gaps in graphene nanoribbons, Phys. Rev. Lett. 97 (2006) 216803.

[5] K.A. Ritter, J.W. Lyding, The influence of edge structure on the electronic properties of graphene quantum dots and nanoribbons, Nat. Mater. 8 (2009) 235.

[6] O. Hod, V. Barone, J.E. Peralta, G.E. Scuseria, Enhanced half-metallicity in edgeoxidized zigzag graphene nanoribbons, Nano Lett. 7 (2007) 2295.

[7] R. Saito, G. Dresselhaus, M.S. Dresselhaus, Physical Properties of Carbon Nanotubes, Imperial College Press, London, 1998.

[8] Y. Sui, B. Yao, L. Xiao, G. Xing, L. Yang, X. Li, X. Li, J. Lang, S. Lv, J. Cao, M. Gao, J. Yang, Effects of $(\mathrm{P}, \mathrm{N})$ dual acceptor doping on band gap and $p$-type conduction behavior of ZnO films, J. Appl. Phys. 113 (2013) 133701.

[9] V.V. Ilyasov, B.C. Meshi, V.C. Nguyen, I.V. Ershov, D.C. Nguyen, Magnetism and transport properties of zigzag graphene nanoribbons/hexagonal boron nitride heterostructures, J. Appl. Phys. 115 (2014) 053708.

[10] V. Ilyasov, V. Nguyen, I. Ershov, D.C. Nguyen, Ab initio study of structural and electronic properties of zigzag graphene nanoribbons on hexagonal boron nitride, J. Struct. Chem. 55 (2014) 191.

[11] D.C. Elias, R.R. Nair, T.M.G. Mohiuddin, S.V. Morozov, P. Blake, M.P. Halsall, A.C. Ferrari, D.W. Boukhvalov, M.I. Katsnelson, A.K. Geim, K.S. Novoselov, Control of graphene's properties by reversible hydrogenation: evidence for graphane, Science 323 (2009) 610

[12] S. Ryu, M.Y. Han, J. Maultzsch, T.F. Heinz, P. Kim, M.L. Steigerwald, L.E. Brus, Reversible basal plane hydrogenation of graphene, Nano Lett. 8 (2008) 4597
[13] L. Yang, C-H. Park, Y.W. Son, M.L. Cohen, S.G. Louie, Quasiparticle energies and band gaps in graphene nanoribbons, Phys. Rev. Lett. 99 (2007) 186801.

[14] A. Yamashiro, Y. Shimio, K. Harigaya, K. Wakabayashi, Spin- and chargepolarized states in nanographene ribbons with zigzag edges, Phys. Rev. B 68 (2003) 193410.

[15] M. Maruyama, K. Kusakabe, Theoretical prediction of synthesis methods to create magnetic nanographite, J. Phys. Soc. Jpn. 73 (2004) 656-663.

[16] M. Ezawa, Peculiar width dependence of the electronic properties of carbon nanoribbons, Phys. Rev. B 73 (2006) 045432

[17] A. Fleurence, R. Friedlein, T. Ozaki, H. Kawai, Y. Wang, Y. Yamada-Takamura, Experimental evidence for epitaxial silicene on diboride thin films, Phys. Rev. Lett. 108 (2012) 245501.

[18] L. Meng, Y. Wang, L. Zhang, S. Du, R. Wu, L. Li, Y. Zhang, G. Li, H. Zhou, W.A. Hofer, H.-J. Gao, Buckled silicene formation on $\operatorname{Ir}(111)$, Nano Lett. 13 (2013) 685.

[19] S. Cahangirov, M. Topsakal, E. Akturk, H. Sahin, S. Ciraci, Two- and onedimensional honeycomb structures of silicon and germanium, Phys. Rev. Lett. 102 (2009) 236804.

[20] M.E. Dávila, L. Xian, S. Cahangirov, A. Rubio, G. Le Lay, Germanene: a novel two-dimensional germanium allotrope akin to graphene and silicene, New J. Phys. 16 (2014) 095002.

[21] M. Modaresi, A. Kakoee, Y. Mogulkoc, M.R. Roknabadi, Effect of external strain on electronic structure of stanene, Comput. Mater. Sci. 1 (2015) 039.

[22] M. Fadaie, N. Shahtahmasebi, M.R. Roknabadi, Effect of external electric field on the electronic structure and optical properties of stanene, Opt. Quantum Electron. 48 (2016) 440.

[23] J.P. Perdew, K. Burke, Generalized gradient approximation made simple, Phys. Rev. Lett. 77 (1996) 3865-3868.

[24] G. Grosso, G.P. Parravicini, Solid State Physics, Academic Press, 2005.

[25] Y.E. Yang, Y.R. Yang, X.H. Yan, Universal optical properties of graphane nanoribbons: a first-principles study, Physica E 44 (2012) 1406.

[26] W. Kohn, L.J. Sham, Self-consistent equations including exchange and correlation effects, Phys. Rev. 140 (1965) 1133-1138.

[27] Y.R. Yang, X.H. Yana, Y. Xiao, Z.H. Guo, The optical properties of onedimensional ZnO: a first-principles study, Chem. Phys. Lett. 446 (2007) 98-102.

[28] Y. Yu-Rong, Y. Xiao-Hong, G. Zhao-Hui, D. Yu-Xiang, Common electronic band gaps and similar optical properties of ZnO nanotubes, Chin. Phys. B 17 (2008) 3433. 